\title{
Unpacking the Black Box of Causality: Learning about Causal Mechanisms from Experimental and Observational Studies
}

\section{Kosuke Imai}

\section{Princeton University}

December 6, 2012

University of Wisconsin, Madison

Center for Education Research 


\section{Project References}

This talk is based on the following papers and software:

- "Unpacking the Black Box of Causality: Learning about Causal Mechanisms from Experimental and Observational Studies." American Political Science Review

- "Identification, Inference, and Sensitivity Analysis for Causal Mediation Effects." Statistical Science

- "A General Approach to Causal Mediation Analysis." Psychological Methods

- "Experimental Designs for Identifying Causal Mechanisms." Journal of the Royal Statistical Society, Series A

- "Identification and Sensitivity Analysis for Multiple Causal Mechanisms: Revisiting Evidence from Framing Experiments." Political Analysis

- "mediation: R Package for Causal Mediation Analysis." The Comprehensive $R$ Archive Network

All of these and other materials are available at http://imai.princeton.edu/projects/mediation 


\section{Identification of Causal Mechanisms}

- Causal inference is a central goal of scientific research

- Scientists care about causal mechanisms, not just about causal effects

- Randomized experiments often only determine whether the treatment causes changes in the outcome

- Not how and why the treatment affects the outcome

- Common criticism of experiments and statistics:

\section{black box view of causality}

- Question: How can we learn about causal mechanisms from experimental and observational studies? 


\section{Goals of the Talk}

Present a general framework for statistical design and analysis of causal mechanisms

(1) Show that the sequential ignorability assumption is required to identify mechanisms even in experiments

(2) Offer a flexible estimation strategy under this assumption

(3) Propose a sensitivity analysis to probe this assumption

(4) Illustrate how to use the open-source software mediation

(3) Propose new experimental designs that do not rely on sequential ignorability

(6) Cover both experiments and observational studies under the same principle 


\section{Causal Mediation Analysis}

- Causal mechanisms as causal pathways

- Graphical representation

Mediator, $M$

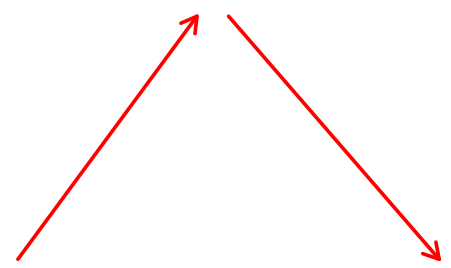

Treatment, $T \longrightarrow$ Outcome, $Y$

- Goal is to decompose total effect into direct and indirect effects

- Example in education research:

- Treatment: New curriculum

- Mediator: Teachers' efforts

- Outcome: Students' test score 


\section{Standard Estimation Methods}

- Standard Linear Structural Equation Models:

$$
\begin{aligned}
Y_{i} & =\alpha_{1}+\beta_{1} T_{i}+\epsilon_{1 i} \\
M_{i} & =\alpha_{2}+\beta_{2} T_{i}+\epsilon_{2 i} \\
Y_{i} & =\alpha_{3}+\beta_{3} T_{i}+\gamma M_{i}+\epsilon_{3 i}
\end{aligned}
$$

- Total effect (ATE) is $\beta_{1}$

- Direct effect is $\beta_{3}$

- Indirect or mediation effect is: $\beta_{2} \gamma$ or $\beta_{1}-\beta_{3}$

- Effect decomposition: $\beta_{1}=\beta_{3}+\beta_{2} \gamma$

- But what are we assuming when we do mediation analysis?

- How sensitive our results are to the violation of this assumption?

- What should we do if our model is not a simple linear regression?

- Are there research designs that require weaker assumptions? 


\section{Media Cues and Immigration Attitudes}

Brader et al. experiment:

- (White) Subjects read a mock news story about immigration

- Treatment: Hispanic immigrant in the story

- Control: European immigrant in the story

- Attitudinal and behavioral outcome variables:

- Opinions about increasing or decrease immigration

- Contact legislator about the issue

- Send anti-immigration message to legislator

- Hypothesis: Hispanic immigrant increases anxiety, leading to greater opposition to immigration 


\section{Causal Mediation Analysis in Brader et al.}

Anxiety, $M$

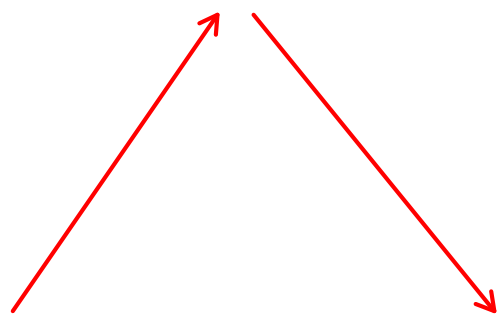

Media Cue, $T \longrightarrow$ Immigration Attitudes, $Y$

What's the effect of the news story that works by making people anxious? 


\section{Potential Outcomes Framework of Causal Inference}

- Binary treatment: $T_{i} \in\{0,1\}$

- Mediator: $M_{i} \in \mathcal{M}$

- Outcome: $Y_{i} \in \mathcal{Y}$

- Observed pre-treatment covariates: $X_{i} \in \mathcal{X}$

- Potential mediators: $M_{i}(t)$, where $M_{i}=M_{i}\left(T_{i}\right)$ observed

- Potential outcomes: $Y_{i}(t, m)$, where $Y_{i}=Y_{i}\left(T_{i}, M_{i}\left(T_{i}\right)\right)$ observed

- Typically, only one potential outcome (mediator) is observed 


\section{Media Cue Study with this Notation}

- $T_{i}=1$ : Adam " $i$ " was (randomly) assigned to the treatment group

- $M_{i}(1)=M_{i}$ : observed level of anxiety reported by Adam

- $M_{i}(0)$ : counterfactual level of Adam's anxiety if assigned to the control condition

- $Y_{i}\left(1, M_{i}(1)\right)=Y_{i}$ : observed immigration attitude reported by Adam

- Suppose Adam got anxious: $M_{i}=M_{i}(1)=1$

- $Y_{i}(1,1)$ : observed outcome

$$
Y_{i}(1,0)
$$

- $\left.\begin{array}{r}Y_{i}(0,1) \\ Y_{i}(0,0)\end{array}\right\}$ counterfactual outcomes 


\section{Causal Mediation Effects}

- Total causal effect:

$$
\tau_{i} \equiv Y_{i}\left(1, M_{i}(1)\right)-Y_{i}\left(0, M_{i}(0)\right)
$$

- Causal mediation (Indirect) effects:

$$
\delta_{i}(t) \equiv Y_{i}\left(t, M_{i}(1)\right)-Y_{i}\left(t, M_{i}(0)\right)
$$

- Causal effect of the change in $M_{i}$ on $Y_{i}$ that would be induced by treatment

- Change the mediator from $M_{i}(0)$ to $M_{i}(1)$ while holding the treatment constant at $t$

- Represents the causal mechanism through $M_{i}$ 


\section{Total Effect $=$ Indirect Effect + Direct Effect}

- Direct effects:

$$
\zeta_{i}(t) \equiv Y_{i}\left(1, M_{i}(t)\right)-Y_{i}\left(0, M_{i}(t)\right)
$$

- Causal effect of $T_{i}$ on $Y_{i}$, holding mediator constant at its potential value that would realize when $T_{i}=t$

- Change the treatment from 0 to 1 while holding the mediator constant at $M_{i}(t)$

- Represents all causal mechanisms other than through $M_{i}$

- Total effect $=$ mediation (indirect) effect + direct effect:

$$
\begin{aligned}
\tau_{i} & =\underbrace{Y_{i}\left(1, M_{i}(1)\right)-Y_{i}\left(1, M_{i}(0)\right)}_{\delta_{i}(1)}+\underbrace{Y_{i}\left(1, M_{i}(0)\right)-Y_{i}\left(0, M_{i}(0)\right)}_{\zeta_{i}(0)} \\
& =\underbrace{Y_{i}\left(1, M_{i}(1)\right)-Y_{i}\left(0, M_{i}(1)\right)}_{\delta_{i}(1)}+\underbrace{Y_{i}}_{Y_{i}\left(0, M_{i}(1)\right)-Y_{i}\left(0, M_{i}(0)\right)}
\end{aligned}
$$




\section{Back to the Media Cue Study}

- Mediation effect: Treatment effect on immigration attitudes that is due to the change in anxiety induced by the treatment news story

- Scenario I: Treatment makes Adam anxious

$\left(M_{i}(1), M_{i}(0)\right)=(1,0)$

- $\delta_{i}(1)=Y_{i}(1,1)-Y_{i}(1,0)$

- $\delta_{i}(0)=Y_{i}(0,1)-Y_{i}(0,0)$

- $\zeta_{i}(1)=Y_{i}(1,1)-Y_{i}(0,1)$

- $\zeta_{i}(0)=Y_{i}(1,0)-Y_{i}(0,0)$

- Scenario II: Adam is always anxious $\left(M_{i}(1), M_{i}(0)\right)=(1,1)$

- $\delta_{i}(1)=Y_{i}(1,1)-Y_{i}(1,1)=0$

- $\delta_{i}(0)=Y_{i}(0,1)-Y_{i}(0,1)=0$

- $\zeta_{i}(1)=Y_{i}(1,1)-Y_{i}(0,1)$

- $\zeta_{i}(0)=Y_{i}(0,1)-Y_{i}(0,1)$

- Zero treatment effect on mediator $\Longrightarrow$ zero mediation effect 


\section{Mechanisms, Manipulations, and Interactions}

\section{Mechanisms}

- Indirect effects: $\delta_{i}(t) \equiv Y_{i}\left(t, M_{i}(1)\right)-Y_{i}\left(t, M_{i}(0)\right)$

- Counterfactuals about treatment-induced mediator values

\section{Manipulations}

- Controlled direct effects: $\xi_{i}\left(t, m, m^{\prime}\right) \equiv Y_{i}(t, m)-Y_{i}\left(t, m^{\prime}\right)$

- Causal effect of directly manipulating the mediator under $T_{i}=t$

\section{Interactions}

- Interaction effects: $\xi\left(1, m, m^{\prime}\right)-\xi\left(0, m, m^{\prime}\right) \neq 0$

- Causal effect of mediator on outcome depends on treatment

- Doesn't imply the existence of a mechanism 


\section{What Does the Observed Data Tell Us?}

- Quantity of Interest: Average causal mediation effects

$$
\bar{\delta}(t) \equiv \mathbb{E}\left(\delta_{i}(t)\right)=\mathbb{E}\left\{Y_{i}\left(t, M_{i}(1)\right)-Y_{i}\left(t, M_{i}(0)\right)\right\}
$$

- Average direct effects $(\bar{\zeta}(t))$ are defined similarly

- Problem: $Y_{i}\left(t, M_{i}(t)\right)$ is observed but $Y_{i}\left(t, M_{i}\left(t^{\prime}\right)\right)$ can never be observed

- Adam received the treatment:

- $Y_{i}\left(1, M_{i}(1)\right)$ is observed

- $Y_{i}\left(0, M_{i}(0)\right)$ is not observed but is potentially observable

- $Y_{i}\left(1, M_{i}(0)\right)$ and $Y_{i}\left(0, M_{i}(1)\right)$ are pure counterfactuals

- We have an identification problem!

$\Longrightarrow$ Need additional assumptions to make progress 


\section{Sequential Ignorability Assumption}

- Proposed identification assumption: Sequential Ignorability (SI)

$$
\begin{aligned}
& \left\{Y_{i}\left(t^{\prime}, m\right), M_{i}(t)\right\} \Perp T_{i} \mid X_{i}=x, \\
& Y_{i}\left(t^{\prime}, m\right) \Perp M_{i}(t) \mid T_{i}=t, X_{i}=x
\end{aligned}
$$

- (1) is guaranteed to hold in a standard experiment

- (2) does not hold unless $X_{i}$ includes all confounders

- $X_{i}$ has to be pre-treatment confounders

- Post-treatment confounder is not allowed $\Longrightarrow$ alternative mediators are allowed but they cannot be causally related (more on this later) 


\section{Nonparametric Identification}

Under SI, both ACME and average direct effects are nonparametrically identified (can be consistently estimated without modeling assumption)

- $\operatorname{ACME} \bar{\delta}(t)$

$$
\iint \mathbb{E}\left(Y_{i} \mid M_{i}, T_{i}=t, X_{i}\right)\left\{d P\left(M_{i} \mid T_{i}=1, X_{i}\right)-d P\left(M_{i} \mid T_{i}=0, X_{i}\right)\right\} d P\left(X_{i}\right)
$$

- Average direct effects $\bar{\zeta}(t)$

$$
\iint\left\{\mathbb{E}\left(Y_{i} \mid M_{i}, T_{i}=1, X_{i}\right)-\mathbb{E}\left(Y_{i} \mid M_{i}, T_{i}=0, X_{i}\right)\right\} d P\left(M_{i} \mid T_{i}=t, X_{i}\right) d P\left(X_{i}\right)
$$

Implies the general "mediation formula" under any model 


\section{Traditional Estimation Method}

- Linear structural equation model (LSEM):

$$
\begin{aligned}
M_{i} & =\alpha_{2}+\beta_{2} T_{i}+\xi_{2}^{\top} X_{i}+\epsilon_{i 2}, \\
Y_{i} & =\alpha_{3}+\beta_{3} T_{i}+\gamma M_{i}+\xi_{3}^{\top} X_{i}+\epsilon_{i 3} .
\end{aligned}
$$

- Fit two least squares regressions separately

- Use product of coefficients $\left(\hat{\beta}_{2} \hat{\gamma}\right)$ to estimate ACME

- Use asymptotic variance to test significance (Sobel test)

- Under SI and the no-interaction assumption $(\bar{\delta}(1) \neq \bar{\delta}(0)), \hat{\beta}_{2} \hat{\gamma}$ consistently estimates ACME

- Can be extended to LSEM with interaction terms

- Problem: Only valid for the simplest LSEM 


\section{Proposed General Estimation Algorithm}

- Model outcome and mediator

- Outcome model: $p\left(Y_{i} \mid T_{i}, M_{i}, X_{i}\right)$

- Mediator model: $p\left(M_{i} \mid T_{i}, X_{i}\right)$

- These models can be of any form (linear or nonlinear, semi- or nonparametric, with or without interactions)

(2) Predict mediator for both treatment values $\left(M_{i}(1), M_{i}(0)\right)$

(3) Predict outcome by first setting $T_{i}=1$ and $M_{i}=M_{i}(0)$, and then $T_{i}=1$ and $M_{i}=M_{i}(1)$

(4) Compute the average difference between two outcomes to obtain a consistent estimate of ACME

(5) Monte-Carlo or bootstrapping to estimate uncertainty 


\section{Example: Binary Mediator and Outcome}

- Two logistic regression models:

$$
\begin{aligned}
\operatorname{Pr}\left(M_{i}=1 \mid T_{i}, X_{i}\right) & =\operatorname{logit}^{-1}\left(\alpha_{2}+\beta_{2} T_{i}+\xi_{2}^{\top} X_{i}\right) \\
\operatorname{Pr}\left(Y_{i}=1 \mid T_{i}, M_{i}, X_{i}\right) & =\operatorname{logit}^{-1}\left(\alpha_{3}+\beta_{3} T_{i}+\gamma M_{i}+\xi_{3}^{\top} X_{i}\right)
\end{aligned}
$$

- Can't multiply $\beta_{2}$ by $\gamma$

- Difference of coefficients $\beta_{1}-\beta_{3}$ doesn't work either

$$
\operatorname{Pr}\left(Y_{i}=1 \mid T_{i}, X_{i}\right)=\operatorname{logit}^{-1}\left(\alpha_{1}+\beta_{1} T_{i}+\xi_{1}^{\top} X_{i}\right)
$$

- Can use our algorithm (example: $\mathbb{E}\left\{Y_{i}\left(1, M_{i}(0)\right)\right\}$ )

(1) Predict $M_{i}(0)$ given $T_{i}=0$ using the first model

(2) Compute $\operatorname{Pr}\left(Y_{i}\left(1, M_{i}(0)\right)=1 \mid X_{i}\right)$ given $T_{i}=1$ and $\widehat{M}_{i}(0)$ using the second model 


\section{Sensitivity Analysis}

- Standard experiments require SI to identify mechanisms

- This assumption is often too strong

- Need to assess the robustness of findings via sensitivity analysis

- Question: How large a departure from the SI assumption must occur for the conclusions to no longer hold?

- Parametric sensitivity analysis by assuming

$$
\left\{Y_{i}\left(t^{\prime}, m\right), M_{i}(t)\right\} \Perp T_{i} \mid X_{i}=x
$$

but not

$$
Y_{i}\left(t^{\prime}, m\right) \Perp M_{i}(t) \mid T_{i}=t, X_{i}=x
$$

- Possible existence of unobserved pre-treatment confounder 


\section{Parametric Sensitivity Analysis}

- Sensitivity parameter: $\rho \equiv \operatorname{Corr}\left(\epsilon_{i 2}, \epsilon_{i 3}\right)$

- Sequential ignorability implies $\rho=0$

- Set $\rho$ to different values and see how ACME changes

- Result:

$$
\bar{\delta}(0)=\bar{\delta}(1)=\frac{\beta_{2} \sigma_{1}}{\sigma_{2}}\left\{\tilde{\rho}-\rho \sqrt{\left(1-\tilde{\rho}^{2}\right) /\left(1-\rho^{2}\right)}\right\},
$$

where $\sigma_{j}^{2} \equiv \operatorname{var}\left(\epsilon_{i j}\right)$ for $j=1,2$ and $\tilde{\rho} \equiv \operatorname{Corr}\left(\epsilon_{i 1}, \epsilon_{i 2}\right)$.

- When do my results go away completely?

- $\bar{\delta}(t)=0$ if and only if $\rho=\tilde{\rho}$

- Easy to estimate from the regression of $Y_{i}$ on $T_{i}$ :

$$
Y_{i}=\alpha_{1}+\beta_{1} T_{i}+\epsilon_{i 1}
$$




\section{Interpreting Sensitivity Analysis with R squares}

- Interpreting $\rho$ : how small is too small?

- An unobserved (pre-treatment) confounder formulation:

$$
\epsilon_{i 2}=\lambda_{2} U_{i}+\epsilon_{i 2}^{\prime} \text { and } \epsilon_{i 3}=\lambda_{3} U_{i}+\epsilon_{i 3}^{\prime}
$$

- How much does $U_{i}$ have to explain for our results to go away?

- Sensitivity parameters: R squares

(- Proportion of previously unexplained variance explained by $U_{i}$

$$
R_{M}^{2 *} \equiv 1-\frac{\operatorname{var}\left(\epsilon_{i 2}^{\prime}\right)}{\operatorname{var}\left(\epsilon_{i 2}\right)} \quad \text { and } \quad R_{Y}^{2 *} \equiv 1-\frac{\operatorname{var}\left(\epsilon_{i 3}^{\prime}\right)}{\operatorname{var}\left(\epsilon_{i 3}\right)}
$$

(2) Proportion of original variance explained by $U_{i}$

$$
\widetilde{R}_{M}^{2} \equiv \frac{\operatorname{var}\left(\epsilon_{i 2}\right)-\operatorname{var}\left(\epsilon_{i 2}^{\prime}\right)}{\operatorname{var}\left(M_{i}\right)} \quad \text { and } \quad \widetilde{R}_{Y}^{2} \equiv \frac{\operatorname{var}\left(\epsilon_{i 3}\right)-\operatorname{var}\left(\epsilon_{i 3}^{\prime}\right)}{\operatorname{var}\left(Y_{i}\right)}
$$


- Then reparameterize $\rho$ using $\left(R_{M}^{2 *}, R_{Y}^{2 *}\right)\left(\operatorname{or}\left(\widetilde{R}_{M}^{2}, \widetilde{R}_{Y}^{2}\right)\right)$ :

$$
\rho=\operatorname{sgn}\left(\lambda_{2} \lambda_{3}\right) R_{M}^{*} R_{Y}^{*}=\frac{\operatorname{sgn}\left(\lambda_{2} \lambda_{3}\right) \widetilde{R}_{M} \widetilde{R}_{Y}}{\sqrt{\left(1-R_{M}^{2}\right)\left(1-R_{Y}^{2}\right)}},
$$

where $R_{M}^{2}$ and $R_{Y}^{2}$ are from the original mediator and outcome models

- $\operatorname{sgn}\left(\lambda_{2} \lambda_{3}\right)$ indicates the direction of the effects of $U_{i}$ on $Y_{i}$ and $M_{i}$

- Set $\left(R_{M}^{2 *}, R_{Y}^{2 *}\right)$ (or $\left.\left(\widetilde{R}_{M}^{2}, \widetilde{R}_{Y}^{2}\right)\right)$ to different values and see how mediation effects change 


\section{Reanalysis: Estimates under Sequential Ignorability}

- Original method: Product of coefficients with the Sobel test

- Valid only when both models are linear w/o $T-M$ interaction (which they are not)

- Our method: Calculate ACME using our general algorithm

Outcome variables

Decrease Immigration

$$
\bar{\delta}(1)
$$

Support English Only Laws

$\bar{\delta}(1)$

Request Anti-Immigration Information

$$
\bar{\delta}(1)
$$

Send Anti-Immigration Message

$$
\bar{\delta}(1)
$$

Product of

Coefficients

.347

$[0.146,0.548]$

.204

$[0.069,0.339]$

.277

$[0.084,0.469]$

.276

[0.102, 0.450]
Average Causal Mediation Effect $(\delta)$ 


\section{Reanalysis: Sensitivity Analysis w.r.t. $\rho$}

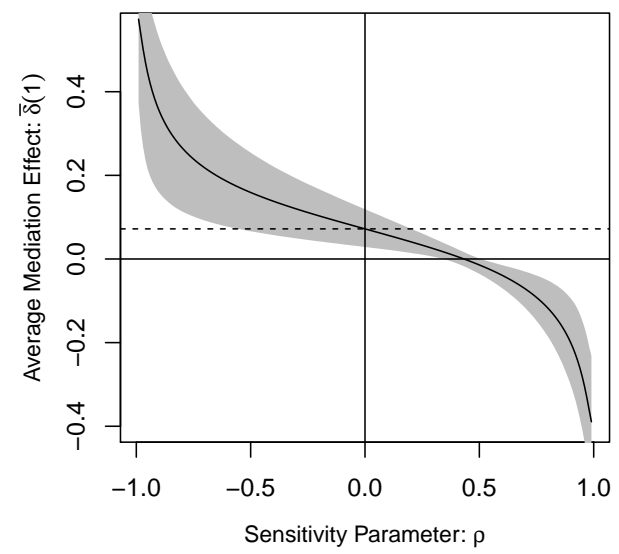

- $\mathrm{ACME}>0$ as long as the error correlation is less than 0.39 (0.30 with $95 \% \mathrm{Cl}$ ) 


\section{Reanalysis: Sensitivity Analysis w.r.t. $\tilde{R}_{M}^{2}$ and $\tilde{R}_{Y}^{2}$}

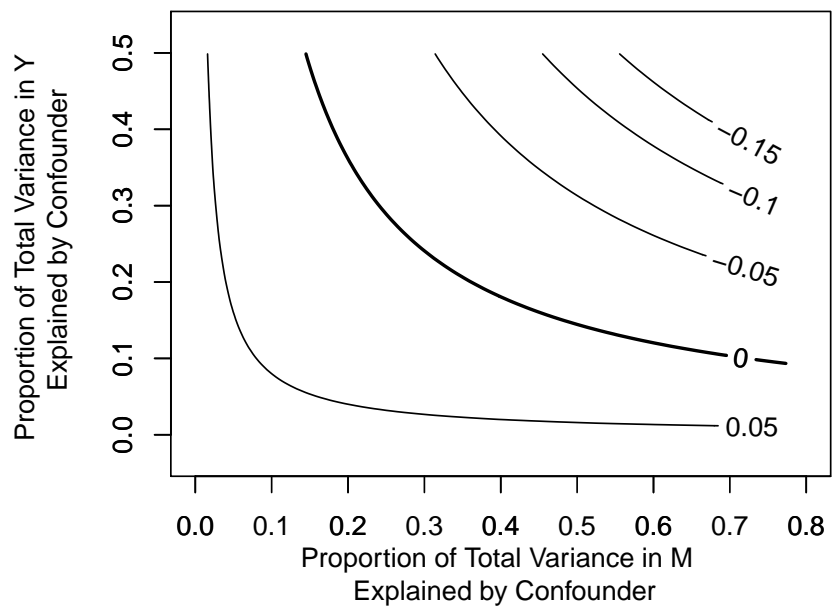

- An unobserved confounder can account for up to $26.5 \%$ of the variation in both $Y_{i}$ and $M_{i}$ before ACME becomes zero 


\section{Overview of $\mathbf{R}$ Package mediation}

Model-Based Inference Design-Based Inference

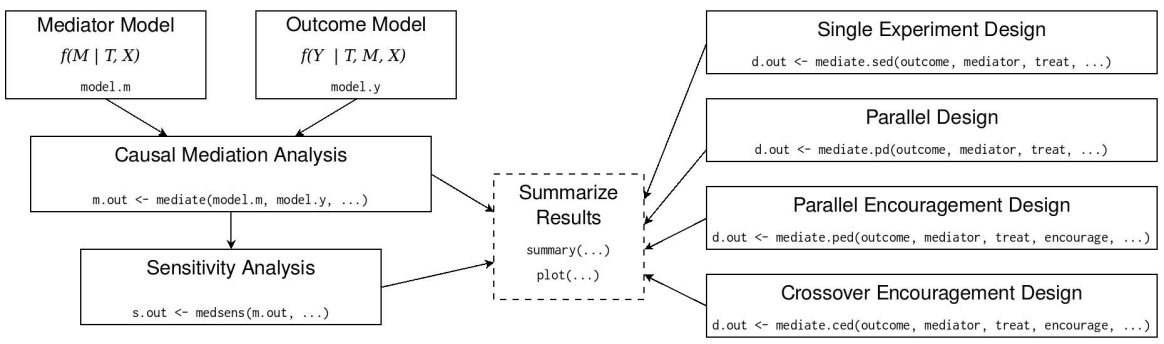

- All of our proposed methods (and more!) can be implemented

- Stata version is also available but has limited capabilities 


\section{Implementation Examples}

(1) Fit models for the mediator and outcome variable and store these models

$>\mathrm{m}<-\operatorname{lm}($ Mediator $\sim$ Treat $+\mathrm{X})$
$>\mathrm{y}<-\operatorname{lm}(\mathrm{Y} \sim$ Treat + Mediator $+\mathrm{X})$

(2) Mediation analysis: Feed model objects into the mediate () function. Call a summary of results

$>$ m.out<-mediate $(\mathrm{m}, \mathrm{y}$, treat = "Treat", mediator = "Mediator")

$>$ summary $(\mathrm{m}$. out $)$

(3) Sensitivity analysis: Feed the output into the medsens () function. Summarize and plot

$>$ s.out <- medsens (m.out)

$>$ summary (s.out)

$>$ plot (s.out, "rho")

$>\operatorname{plot}(\mathrm{s}$. out, $" \mathrm{R} 2 ")$ 


\section{Beyond Sequential Ignorability}

- Without sequential ignorability, standard experimental design lacks identification power

- Even the sign of ACME is not identified

- Need to develop alternative experimental designs for more credible inference

- Possible when the mediator can be directly or indirectly manipulated

- All proposed designs preserve the ability to estimate the ACME under the SI assumption

- Trade-off: statistical power 


\section{Parallel Design}

\begin{tabular}{|l|l|}
\hline \multicolumn{1}{|c|}{$\begin{array}{l}\text { Randomly } \\
\text { split sample }\end{array}$} \\
$\begin{array}{ll}\text { Experiment 1 } \\
\text { treatment }\end{array}$ & $\begin{array}{l}\text { Experiment 2 } \\
\text { 1) Randomize } \\
\text { treatment }\end{array}$ \\
\begin{tabular}{ll} 
2) Measure mediator & 2) Randomize mediator \\
\hline
\end{tabular}
\end{tabular}

- Must assume no direct effect of manipulation on outcome

- More informative than standard single experiment

- If we assume no $T-M$ interaction, ACME is point identified 


\section{Why Do We Need No-Interaction Assumption?}

- Numerical Example:

\begin{tabular}{c|cccc|c}
\hline Prop. & $M_{i}(1)$ & $M_{i}(0)$ & $Y_{i}(t, 1)$ & $Y_{i}(t, 0)$ & $\delta_{i}(t)$ \\
\hline 0.3 & 1 & 0 & 0 & 1 & -1 \\
0.3 & 0 & 0 & 1 & 0 & 0 \\
0.1 & 0 & 1 & 0 & 1 & 1 \\
0.3 & 1 & 1 & 1 & 0 & 0 \\
\hline
\end{tabular}

- $\mathbb{E}\left(M_{i}(1)-M_{i}(0)\right)=\mathbb{E}\left(Y_{i}(t, 1)-Y_{i}(t, 0)\right)=0.2$, but $\bar{\delta}(t)=-0.2$

- The Problem: Causal effect heterogeneity

- $T$ increases $M$ only on average

- $M$ increases $Y$ only on average

- $T-M$ interaction: Many of those who have a positive effect of $T$ on $M$ have a negative effect of $M$ on $Y$ (first row)

- Pitfall of "mechanism experiments" or "causal chain approach" 


\section{Example from Behavioral Neuroscience}

Why study brain?: Social scientists' search for causal mechanisms underlying human behavior

- Psychologists, economists, and even political scientists

Question: What mechanism links low offers in an ultimatum game with "irrational" rejections?

- A brain region known to be related to fairness becomes more active when unfair offer received (single experiment design)

Design solution: manipulate mechanisms with TMS

- Knoch et al. use TMS to manipulate - turn off - one of these regions, and then observes choices (parallel design) 


\section{Encouragement Design}

- Direct manipulation of the mediator is often impossible

- Even if possible, manipulation can directly affect outcome

- Need to allow for subtle and indirect manipulations

- Randomly encourage subjects to take particular values of the mediator $M_{i}$

- Standard instrumental variable assumptions (Angrist et al.)

Use a $2 \times 3$ factorial design:

(1) Randomly assign $T$

(2) Also randomly decide whether to positively encourage, negatively encourage, or do nothing

(3) Measure mediator and outcome

- Informative inference about the "complier" ACME

- Reduces to the parallel design if encouragement is perfect 


\section{Crossover Design}

- Recall ACME can be identified if we observe $Y_{i}\left(t^{\prime}, M_{i}(t)\right)$

- Get $M_{i}(t)$, then switch $T_{i}$ to $t^{\prime}$ while holding $M_{i}=M_{i}(t)$

- Crossover design:

(1) Round 1: Conduct a standard experiment

(2) Round 2: Change the treatment to the opposite status but fix the mediator to the value observed in the first round

- Very powerful - identifies mediation effects for each subject

- Must assume no carryover effect: Round 1 doesn't affect Round 2

- Can be made plausible by design 


\section{Example from Labor Economics}

Bertrand \& Mullainathan (2004, AER)

- Treatment: Black vs. White names on CVs

- Mediator: Perceived qualifications of applicants

- Outcome: Callback from employers

- Quantity of interest: Direct effects of (perceived) race

- Would Jamal get a callback if his name were Greg but his qualifications stayed the same?

- Round 1: Send Jamal's actual CV and record the outcome

- Round 2: Send his CV as Greg and record the outcome

- Assumptions can be made plausible 


\section{Designing Observational Studies}

- Key difference between experimental and observational studies: treatment assignment

- Sequential ignorability:

- Ignorability of treatment given covariates

(2) Ignorability of mediator given treatment and covariates

- Both (1) and (2) are suspect in observational studies

- Statistical control: matching, propensity scores, etc.

- Search for quasi-randomized treatments: "natural" experiments

- How can we design observational studies?

- Experiments can serve as templates for observational studies 


\section{Cross-Over Design Examples from Political Science}

EXAMPLE Incumbency advantage

- Estimation of incumbency advantages goes back to 1960 s

- Why incumbency advantage? Scaring off quality challenger

- Use of cross-over design (Levitt and Wolfram)

( ) 1st Round: two non-incumbents in an open seat

(2) 2nd Round: same candidates with one being an incumbent

- Assume challenger quality (mediator) stays the same

- Estimation of direct effect is possible

- Redistricting as natural experiments (Ansolabehere et al.)

(1) 1st Round: incumbent in the old part of the district

(2) 2nd Round: incumbent in the new part of the district

- Challenger quality is the same but treatment is different

- Estimation of direct effect is possible 


\section{Concluding Remarks}

- Even in a randomized experiment, a strong assumption is needed to identify causal mechanisms

- However, progress can be made toward this fundamental goal of scientific research with modern statistical tools

- A general, flexible estimation method is available once we assume sequential ignorability

- Sequential ignorability can be probed via sensitivity analysis

- More credible inferences are possible using clever experimental designs

- Insights from new experimental designs can be directly applied when designing observational studies 
The project website for papers and software:

http://imai.princeton.edu/projects/mechanisms.html

Email for comments and suggestions:

kimai@Princeton.Edu 